\title{
Ein Plädoyer für kritische Perspektiven in der qualitativen Forschung
}

\author{
Rainer Winter
}

Keywords: Krise der Repräsentation; Validität; Ethnografie; Performanz; soziale Gerechtigkeit
Zusammenfassung: Die Herausbildung eines kritischen Poststrukturalismus in den USA hat die zunächst in der Anthropologie diagnostizierte "Krise der Repräsentation" auch in der qualitativen Forschung zu einem zentralen Problem gemacht. Dies führte zu einer Dekonstruktion der Grundlagen der traditionellen Sozialforschung, zur Berücksichtigung ethischer Fragestellungen und zur Suche nach neuen Formen der Validität.

Vor diesem Hintergrund werden in diesem Beitrag verschiedene Methoden und Forschungsstrategien einer kritischen qualitativen Forschung vorgestellt und untersucht. Es sind dies der interpretative Interaktionismus, die Autoethnografie und die performance ethnography. Dabei wird auch der damit verbundene Aufruf zum Engagement, der zu kulturellen und gesellschaftlichen Veränderungen beitragen möchte, diskutiert.

\section{Inhaltsverzeichnis}

1. Qualitative Forschung und soziale Gerechtigkeit

2. Kritischer Poststrukturalismus und das Problem der Repräsentation

3. Strategien und Methoden einer kritischen qualitativen Forschung

3.1 Der interpretative Interaktionismus

3.2 Die Autoethnografie

3.3 Performance ethnography

4. Schluss

Literatur

Zum Autor

Zitation

\section{Qualitative Forschung und soziale Gerechtigkeit}

Zygmunt BAUMAN (2005, S.1097) stellt in seinen Überlegungen zur "flüchtigen Moderne" fest, dass es die Wahl zwischen einer "neutralen" und einer "engagierten" Weise, Soziologie im 21. Jahrhundert zu betreiben, nicht gebe. Es stelle eine Flucht vor der Verantwortung für die Folgen der eigenen wissenschaftlichen Tätigkeit dar, wenn man die Auffassung hegt, man könne einen distanzierten, unabhängigen und objektiven Beobachter/innenstandpunkt einnehmen. Seiner Auffassung nach ist es heute die Aufgabe der Forschung, Möglichkeiten aufzuzeigen, wie wir mit wenig oder ohne Leid zusammenleben können. [1]

Um dieses Leiden der Welt und an der Welt zu reduzieren oder abzuschaffen, müssen wir - so meine Überzeugung - eine Demokratie entwickeln, die, wie Ulrich BECK (2007) fordert, mehr als eine "Expertokratie" darstellt. Sie muss radikal, partizipatorisch und global sein (vgl. WINTER 2010). Für Cornelius CASTORIADIS (1984) ist erst eine wahrhaft demokratische Gesellschaft 
autonom, eine Gesellschaft, die sich ihrer eigenen Gestaltungsmöglichkeiten bewusst ist, die alles Vorgegebene und ihre eigenen Voraussetzungen infrage stellen und die Schaffung neuer Bedeutungen ins Zentrum rücken kann. Die Freiheit der Individuen besteht dann gerade darin, den Sinn schaffen und realisieren zu können, den sie in ihrem Leben für angemessen und nützlich halten. [2]

Die Soziologie soll das oft unsichtbare Elend aufdecken, das Pierre BOURDIEU und sein Forschungsteam (1997) sichtbar zu machen versucht haben. Nur im Kampf gegen dieses Unglück kann das Potenzial menschlicher Freiheit realisiert werden. Auch das von Richard RORTY (1989) angeführte Begehren nach Solidarität, das uns die Möglichkeit gibt, unsere Tätigkeit in einer Gemeinschaft reflexiv zu verankern, lässt sich hier anführen. [3]

Vor diesem Hintergrund möchte ich im Folgenden für ein kritisches Verständnis qualitativer Forschung plädieren. Diese soll durch transformative und interventionistische Untersuchungen eine emanzipatorische Agenda zur Verwirklichung sozialer Gerechtigkeit entwickeln sowie zu einer radikalen Demokratie beitragen. Die qualitative Forschung kann Phänomene und Probleme aus verschiedenen Perspektiven beschreiben und so alternative Sicht- und Denkweisen entwickeln. Nicht nur die Soziologie, sondern auch die Psychologie und Pädagogik, ja alle Sozial- und Geisteswissenschaften sollen ihren Beitrag zur kritischen Konversation über Demokratie, Geschlechtsrollen, kulturelle sowie soziale Ungleichheit, Freiheit und Gemeinschaft leisten (vgl. DENZIN \& LINCOLN 2005, S.3). Eine kritische qualitative Forschung möchte Veränderungen im Alltagsleben von Menschen bewirken. Es sollen Differenzen, die von Bedeutung sind und neue Perspektiven und Konstruktionen erlauben, erzeugt sowie Handlungsmöglichkeiten und alternative Formen des Zusammenlebens eröffnet werden. Angesichts der Allgegenwart von kriegerischen und terroristischen Auseinandersetzungen, angesichts der Hegemonie des Neoliberalismus in unserer Zeit und angesichts der realen Subsumtion aller gesellschaftlichen Bereiche unter Prozesse der Kapitalverwertung soll qualitative Forschung nicht nur die negativen Folgen dieses kulturellen und gesellschaftlichen Wandels aufzeigen - etwa die sich verstärkenden Formen sozialer und kultureller Ungleichheit, die in Deutschland den Begriff der Klassengesellschaft wieder sinnvoll machen - sondern auch eine "Politik des Möglichen", die die Hoffnung auf demokratische Veränderungen und utopische Visionen bewahrt (vgl. KELLNER 1995). Die sich herausbildende globale Zivilgesellschaft benötigt eine Gemeinschaft von Forschern und Forscherinnen, die diesen Prozess begleitet, unterstützt und voranbringt. [4]

Ich werde zunächst zeigen, wie durch die Herausbildung eines kritischen Poststrukturalismus die zunächst in der Anthropologie diagnostizierte Krise der Repräsentation auch in der qualitativen Forschung in den USA zu einem zentralen Problem wurde. Dies führte zu einer Dekonstruktion der Grundlagen der traditionellen Sozialforschung, zur Berücksichtigung ethischer Fragestellungen und zur Suche nach neuen Formen der Validität (Abschnitt 2). Anschließend werde ich verschiedene Methoden und Forschungsstrategien einer 
kritischen qualitativen Forschung vorstellen und diskutieren. Es sind dies der interpretative Interaktionismus, die Autoethnografie und die performance ethnography (Abschnitt 3). Dabei diskutiere ich den damit verbundenen Aufruf zum Engagement, der zu kulturellen und gesellschaftlichen Veränderungen beitragen möchte. Ein kurzes Fazit beendet meine Ausführungen (Abschnitt 4). [5]

\section{Kritischer Poststrukturalismus und das Problem der Repräsentation}

Ein wichtiger Ausgangspunkt der kritischen qualitativen Forschung ist die vor allem in Auseinandersetzung mit der Kritik der Präsenzmetaphysik von Jacques DERRIDA und mit Michel FOUCAULTs Diskursanalyse entwickelte Annahme, dass gelebte Erfahrung nicht direkt wiedergegeben oder repräsentiert werden kann. Dies bedeutet nicht das Ende der Repräsentation, aber das Aufgeben der Vorstellung einer "reinen Präsenz". Gerade DERRIDA (1988) konstatiert eine "Unausweichlichkeit der Repräsentation". Zentral ist nun nicht mehr die Verantwortung dafür, Sachverhalte in sich selbst dazustellen, sondern es geht darum, das Netz von Struktur, Zeichen und Spiel sozialer Beziehungen, in das wir eingebunden sind, sichtbar zu machen. So ist das Ziel nicht eine genauere Repräsentation, sondern zu untersuchen, welche Rahmen, verstanden als Räume konstruierter Sichtbarkeit und als Diskurse der Anreizung, unser Sehen bestimmen und wie sie transformiert werden können (LATHER 2001 [1993], S.31f.). [6]

Daher kann es keinen direkten Zugang zur Innenwelt der Untersuchten geben.. Beobachtungen können nicht "objektiv" sein, sondern sind immer sozial in den Welten der Untersuchten und Untersuchenden lokalisiert (vgl. DENZIN \& LINCOLN 2005a, S.21). Die gelebte Erfahrung ist zudem bereits durch Texte sowie Diskurse geprägt und strukturiert, sodass wir auch von einer gelebten Textualität sprechen können, die die Erfahrungen und Praktiken durchdringt. So spiegeln Sprache, Diskurse und Texte nicht Erfahrungen wider, sondern schaffen sie neu. In diesem kreativen Prozess wird das, was beschrieben wird, moduliert, transformiert und aufgeschoben. Es gibt Sprünge und Lücken zwischen der Realität, der Erfahrung und ihrer Artikulation (BRUNER 1986). "There can never be a final, accurate representation of what was meant or said - only different textual representations of different experiences" (DENZIN 1997, S.5). [7]

Diese Auffassung hat weitreichende Folgen für den Prozess einer qualitativen Sozialforschung. Eine wesentliche Implikation ist, dass die Erfahrungen und Perspektiven, die in den Texten von qualitativen Forschern bzw. Forscherinnen beschrieben und analysiert werden, keine unabhängige Wirklichkeit wiedergeben, sondern durch diesen Prozess des Forschens und Schreibens hervorgebracht werden. Es sind die materiellen Praktiken der Repräsentation, die die Welt und die Erfahrungen zugänglich machen und uns auf diese Weise auch eine Kenntnis von den Anderen vermitteln. "These practices transform the world. They turn the world into a series of representations, including field notes, interviews, conversations, photographs, recordings, and memos of the self" (DENZIN \& LINCOLN 2005a, S.3). Jede Praktik macht die Welt in einer anderen Weise 
sichtbar. So entstehen verschiedene textuelle Repräsentationen verschiedener Erfahrungen. [8]

Selbstreflexiv wird der bereits aus der Ethnopsychoanalyse (vgl. DEVEREUX 1973) bekannte Zusammenhang eingestanden, dass die Repräsentation der Untersuchten eng mit der Selbstpräsentation des Forschers/der Forscherin verknüpft ist (DENZIN 2009, S.91). Es geht um die Bilder von den Anderen, die sich Forschende machen und die immer auch auf sie verweisen. Eine poststrukturalistische Sensibilität animiert die Forschenden dazu, sich selbst in ihren Forschungstexten zu thematisieren und einzubringen. Schreiben wird im Sinne von Laurel RICHARDSON (2003) zu einer kreativen analytischen Praktik, zu einem schöpferischen Akt der Enthüllung und der Forschung. Das Selbst wird im Schreiben und durch das Schreiben des Textes konstituiert, in seinen Beziehungen dargestellt und in Szene gesetzt. [9]

In diesem Zusammenhang hat RICHARDSON auch vorgeschlagen, den Begriff der Triangulation durch den der Kristallisierung zu ersetzen.

"Rather, the central imaginary is the crystal, which combines symmetry and substance with an infinite variety of shapes, substances, transmutations, multidimensionalities, and angles of approach. Crystals grow, change, alter, but are not amorphous. Crystals are prisms that reflect externalities and refract within themselves. What we see depends upon our angle of repose" (S.517). [10]

So wird zum Ausdruck gebracht, wie sich die Sicht der Wirklichkeit je nach Perspektive und methodologischem Vorgehen verändert. Wirklichkeit kann nicht von außen "objektiv" beschrieben werden, vielmehr sind Forschung und Wirklichkeit unauflöslich miteinander verschränkt. In der Interaktion von Forscher/in und Untersuchten wird die Wirklichkeit geschaffen, über die geforscht wird. Ontologisch betrachtet, wird sie deshalb als veränderbar und prozesshaft begriffen, erkenntnistheoretisch sind die untersuchten Wirklichkeiten soziale Konstruktionen der Forschung. Die traditionelle Vorstellung, es gebe eine privilegierte Weise, auf die Welt zu schauen, man denke, an den Blick des "weißen Mannes" oder an den "wertfreien Wissenschaftler" in der Tradition von Max WEBER, wird aufgegeben. Stattdessen geht es darum, verschiedene Perspektiven auf die Wirklichkeit zu fabrizieren, plurale Wirklichkeiten und vor allem alternative Sicht- und Schreibweisen, die den (bisher) akzeptierten und dominanten wissenschaftlichen "Wahrheiten" widersprechen. Auf diese Weise können multiple "Wahrheiten" hervorgebracht werden, die untereinander auch inkommensurabel sein können. In einem Prozess der Kristallisation kann dieselbe Geschichte aus verschiedenen Perspektiven oder mit unterschiedlichen Textsorten erzählt werden, die z.B. wissenschaftliche mit literarischen Texten, Feldnotizen mit Gedichten mischen. Die unterschiedlichen Wirklichkeiten sollen nicht nur verstanden und je nach Textsorte emphatisch nachvollzogen, sondern auch zueinander in Beziehung gesetzt werden. [11] 
symbolische Konstruktionen der Welt. Vielmehr wird die Forschung als eine Kraft betrachtet, die die Wirklichkeit in symbolischer und materieller Hinsicht transformiert. So zeigt etwa die Geschichte der Psychiatrie, wie Forschung und Wissenschaft problematische und repressive Welten schaffen können, die materiell in Praktiken und symbolisch in Diskursen verankert sind. Auch in dieser Sichtweise ist Forschung nie "objektiv", sondern eine materiell-semiotische Kraft, die eine gesellschaftliche oder politische Agenda mit entsprechenden Dispositiven impliziert. Die Voraussetzung für Interventionen ist eine Analyse der miteinander verschränkten kulturellen, politischen, ökonomischen und ökologischen Prozesse. In dieser kritisch poststrukturalistischen Richtung geht die Wirklichkeit nicht in Konstruktionen auf, sondern sie kann auch "zurückschlagen". Die soziale und materielle Umgebung ermöglicht Forschung, kann sich ihr aber auch entziehen. [12]

Die Krise der Repräsentation mündet zwangsläufig in eine Krise der Legitimation, weil traditionelle Vorstellungen von Validität, die eine Forschung in Bezug auf ihre Korrespondenz zur Wirklichkeit bewerten, problematisiert, infrage gestellt und verabschiedet werden. Im Positivismus wird davon ausgegangen, dass die untersuchten Phänomene "objektiv" dargestellt werden können und so die Wahrheit sichtbar wird. Dieser naive und direkte Realismus hat seine Glaubwürdigkeit verloren. Auch die postpositivistische Version der Validität, die eine Reihe von Regeln vorsieht, wie man sich auf die Wirklichkeit außerhalb des Textes beziehen solle und "Wahrheit" hervorgebracht werden könne, wird im kritischen Poststrukturalismus abgelehnt, weil sie ebenfalls noch der Vorstellung anhängt, eine "Welt da draußen" könne wahrheitsgemäß und akkurat dargestellt werden. Im kritischen Realismus des Postpositivismus gelten für Forschungsprojekte nämlich Regeln und Prozeduren, die bei erfolgreicher Einhaltung bzw. Durchführung diese in der Wirklichkeit verankern und die in Anspruch genommene Autorität, diese wahrheitsgemäß darzustellen, legitimieren. Die Validität verbürgt die Autorität der Forscherin bzw. des Forschers und inauguriert ein partikulares Wahrheitssystem (vgl. DENZIN 1997; LATHER 2003 [1986]). [13]

Dagegen verzichtet der kritische Poststrukturalismus auf diese Grundlegungen und lehnt traditionelle Kriterien zur Bewertung von Forschungsergebnissen ab. Keine Methode oder Theorie hat einen universalen Anspruch auf "Wahrheit" oder auf Wissen, das mit endgültiger Autorität verbunden ist. Validität wird vielfältig, partiell und endlos aufgeschoben. Forschungspraktiken - wie z.B. das Interviewen oder Beobachten - stellen Handlungsformen dar, die partikulare, situierte Formen des Verstehens hervorbringen. Sie machen flüchtige Augenblicke bzw. Ausschnitte der prozesshaften Wirklichkeit einsehbar. Relevant ist in dieser Perspektive jede an einem besonderen Ort und zu einer besonderen Zeit untersuchte Praktik. Sie stellt etwa in der Form des Erzählens einer Geschichte eine partikulare Aufführung und eine Reihe von wechselnden, miteinander in Konflikt stehenden Bedeutungen dar (vgl. FISKE 1999). Dabei können die in einem spezifischen Kontext auftretenden Praktiken nicht einfach auf andere Kontexte generalisiert werden. Vielmehr sollen sie in ihrer unhintergehbaren Singularität beschrieben, analysiert und diagnostiziert werden. [14] 
Was kann nun unter einer poststrukturalistischen Form der Legitimation von Forschungsergebnissen verstanden werden? Ein im traditionellen Sinne valider und mit Autorität versehener Forschungstext, weil er in Korrespondenz zur Wirklichkeit steht, wird aus erkenntnistheoretischen Gründen problematisiert und schlussendlich abgelehnt. Stattdessen werden verschiedene Formen alternativer Validität entwickelt, die politisch und ethisch verankert sind. Einige davon möchte ich kurz vorstellen. Grundsätzlich geht es um die Entfaltung demokratischer, partizipatorischer und emanzipatorischer Perspektiven. [15]

Ein Forschungstext soll z.B. Macht- und Herrschaftsverhältnisse aufdecken und darlegen, wie sie das postmoderne Leben prägen. So kann eine Diskursanalyse zeigen, wie operierende Diskurse das Spiel von Möglichkeiten einschränken und zu scheinbar unverrückbaren Festlegungen und Fixierungen führen. Im Sinne von Patti LATHER (2003 [1986]) erreicht ein Forschungstext z.B. dann eine "katalytische Validität", wenn er einer Gemeinschaft von Forschern und Forscherinnen hilft, derartig strukturierte Verhältnisse besser zu verstehen, sie gesellschaftlich zu kontextualisieren und zu verändern. Es geht darum, eine Ermächtigung der Erforschten, die zu Ko-Forschenden werden sollen, und auch der Forscher und Forscherinnen herbeizuführen. Durch den Forschungsprozess sollen Wissen und Macht demokratisiert werden. Die kritische Pädagogik von Paolo FREIRE und ihr Programm der kulturellen Transformation durch Zusammenarbeit und Dialog haben diesen Ansatz inspiriert (vgl. McLAREN 2001; WINTER 2006; ZIMA 2007). [16]

Jede Form des Verstehens beruht im Sinne des Poststrukturalismus - und auch der Hermeneutik - auf einer "Voreingenommenheit", verankert in einem für selbstverständlich gehaltenen Wissen und in Diskursen. Deshalb soll im Forschungsprozess aufgedeckt werden, was für "wahr" gehalten wird. So soll nach Norman DENZIN (1991, 1997, S.13) ein Forschungstext angesichts der medial konstituierten Hyperrealität der Gegenwart, dem von Jean BAUDRILLARD (1982, 1983) konstatierten postmodernen Exzess, die unterschiedlichen Wahrheitsregimes und vielfältigen Simulationen, die das Reale strukturieren, in ihrer Komplexität und Vielschichtigkeit untersuchen und auf diese Weise eine dekonstruktive Plausibilität bei den Lesenden erreichen soll. Durch die Analyse der pluralen Rahmen, in denen ein besonderes Phänomen verstanden werden kann, wird die Kontingenz jedes Verstehens sichtbar. FOUCAULTs Genealogie und interpretativer Analytik folgend, werden scheinbar universale und zeitlose Ideen und "Wahrheiten" als Effekte spezifischer historischer und sozialer Dispositive ausgewiesen. Dann wird untersucht, welche sozialen und politischen Projekte mit den "Wahrheiten" verbunden sind. Schließlich soll in einer dekonstruktiven Kritik die binäre Logik bestimmt werden, die unser Verständnis partikularer Phänomene prägt. Zusammenfassend lässt sich sagen, dass eine dekonstruktive Validität dann erreicht werden kann, wenn der historische, soziale und politische Charakter sozialer "Wahrheiten" und ihrer Effekte bestimmt wird. Gleichzeitig müssen die Voraussetzungen und das Engagement der eigenen Forschung reflektiert werden. [17] 
Auch die von der Hermeneutik geprägte dialogische Validität basiert auf der Selbstreflexivität der Forschenden, die die Diskurse, die ihre Wahrnehmung der Welt rahmen, problematisieren, sich ihrer bewusst werden und nach Alternativen suchen. Kooperative Formen der Forschung, in die die Untersuchten sich einbringen können, untermauern den dialogischen Charakter. Dabei ist auch hier zu beachten, dass gelebte Wirklichkeiten vielfältig sind. Jeder Forscher/jede Forscherin hat es stets mit mehreren Wirklichkeiten zu tun, die einander auch widersprechen können. Diese Polyvokalität des Feldes soll angemessen berücksichtigt werden. Sie bereichert ethnografische Texte, weil Phänomene aus verschiedenen Blickwinkeln konstruiert werden. So wird deutlich, dass eine einzelne gelebte Erfahrung nicht die "Wahrheit" eines Phänomens vermitteln kann. Erst die solide Berücksichtigung unterschiedlicher Perspektiven bildet die Voraussetzung für einen Text, der die differenzierten Verschränkungen persönlicher, interpersonaler und politischer Ebenen erfassen kann. Insgesamt gesehen geht es also darum, die Lebenswelten der untersuchten Personen in ihrer Komplexität und ihren vielfältigen "Wahrheiten" darzustellen. Forschende nehmen nicht die Position "objektiver" Beobachter/innen ein, die ein Geschehen von außen betrachten. In der Interaktion zwischen ihren Welten und denen der Erforschten spielt sich der auf Kooperation aufbauende Forschungsprozess ab. So ist mit der dialogischen Validität auch die ethische Verpflichtung verbunden, den untersuchten Welten gerecht zu werden. [18]

Diese Formen von Validität folgen demokratischen, partizipatorischen und egalitären Vorstellungen. Sie möchten die vielen im Feld vorhandenen Stimmen zu Wort kommen lassen, insbesondere die der Marginalisierten, Ausgegrenzten und (bisher) Ungehörten (vgl. WINTER 2001a). Dominante Diskurse in einer Gesellschaft, die Machtressourcen mobilisieren und sich als universal ausgeben, werden infrage gestellt. Forschende, die diese neuen Formen von Validität favorisieren, möchten alternative Wirklichkeiten konstruieren und vermitteln, die neue Perspektiven und Handlungsmöglichkeiten eröffnen. Hier schließt die kontextuelle Validität an, die dann erreicht werden kann, wenn die existierenden Formen kultureller und sozialer Ungleichheit in einer Gesellschaft bestimmt werden, um den Forschungsprozess entsprechend positionieren und seine soziale Verankerung sensibel und differenziert analysieren zu können. [19]

Die bisherige Diskussion zeigt, dass es keinen privilegierten und von allen geteilten Zugang zur "Wahrheit" geben kann. Es gibt nicht einen einzigen Standpunkt, von dem aus eine endgültige Version der Welt verfasst werden kann, wie Dorothy SMITH (2004 [1974]) feststellt. Die Krise der Repräsentation öffnet jedoch Forschungstexte für neue Stimmen. So gewinnen die Diskurse von ethnischen Minderheiten, Schwulen und Lesben, von Migrant/innen, Arbeitslosen und Armen an Bedeutung. Sie sind in deren Lebenswelten verankert und werden als interpretative Epistemologien verstanden, die kontextuell verankerte Erfahrungen ausdrücken (vgl. NIEDERER 2009). In der jeweiligen Standpunktperspektive soll ein Wissen elaboriert und artikuliert werden, das die dominanten Diskurse und Praktiken umgeht und die Welt aus der Perspektive der Betroffenen zeigt. Da es keinen archimedischen Punkt gibt, von dem aus die Welt gewusst werden kann, sind alle Formen des Wissens partiell, partikular und 
sozial situiert. Jede Sichtweise ist notwendigerweise unvollständig. Sie verweist auf ein Leben innerhalb von Grenzen und Widersprüchen. Auf diese Weise entstehen situierte Geschichten, die existierende Formen kultureller und sozialer Ungleichheit reflektieren und infrage stellen. [20]

Die Unmöglichkeit von Objektivität verleiht zudem jeder Forschung einen politischen Charakter. Wie HARAWAY (1997) hervorhebt, verdeutlicht dies, dass wir für die Wirklichkeiten, Netzwerke und Wesen, die wir hervorbringen, ethisch verantwortlich sind. Zusammen mit Sandra HARDING (2004 [1988]) fordert sie, dass systematisch verschiedene Perspektiven eines untersuchten Phänomens, insbesondere die von unterworfenen und marginalisierten Gruppen, zusammengebracht, verglichen und kritisch bewertet werden. So kann eine "starke Objektivität" erreicht werden. Ein Standpunkt-Text soll die Strukturen der Unterdrückung aufzeigen, für Dorothy SMITH (2004 [1974]) etwa die im Leben von Frauen. Er soll kritisch reflexiv sein und verschiedenen Stimmen Gehör verschaffen. Vor allem die Unterdrückten sollen ihre Rahmungen der Wirklichkeit artikulieren. [21]

Diese bauen aber bereits auf Repräsentationen von Erfahrungen, auf Texten und Diskursen auf. Dennoch ist die gelebte Erfahrung das Fundament der Standpunkt-Epistemologien, als textuelle Form aber stellt sie eine Fiktion dar, eine Konstruktion realistischen Denkens (vgl. DENZIN 1997, S.85). Vor allem die vietnamesische Filmemacherin TRINH T. Minh-ha (1989) hat in ihren Texten und Filmen gezeigt, wie die gelebte Erfahrung von einer Reihe von widersprüchlichen, sich verändernden und flüchtigen Bedeutungen geprägt wird. [22]

Dieser Befund wird durch andere Studien gestützt. So zeigt z.B. Carol R. RONAI in ihrer Autoethnografie des Stripteasetanzens (1998, S.411), dass sowohl die Identität der Tänzerin als auch die der Forscherin nicht eindeutig bestimmbar sind. Ihre endgültigen Bedeutungen werden permanent aufgeschoben, kommen nicht in der Gegenwart an und lassen sich nicht fixieren. Sobald über sie nachgedacht wird, verschwinden sie in einem Strudel von Bedeutungen, Markierungen und Spuren (RONAI 1999). Jede Verwendung von Worten und Bedeutungen greift zwangsläufig auf andere zurück. In ihrer Forschungsarbeit versucht RONAI daher, die beiden Identitäten zueinander in Beziehung zu setzen und das binäre Konstrukt Tänzerin/Forscherin zu unterminieren. Dabei wird die (endgültige) Bestimmung der Differenz zwischen den Identitäten immer auf eine spätere Zeit verschoben (RONAI 1998, S.419). Die Dekonstruktion ihrer Identität als Forscherin ist für RONAI die Voraussetzung für das Erleben von Ambiguität, die zu neuen Impressionen und Erfahrungen führt. Ihre Studie macht auch deutlich, dass es in einer poststrukturalistisch orientierten Ethnografie weniger um Beschreibung als um Momente der "Einschreibung" geht, in denen Forschende eine situierte Version der untersuchten Welt schaffen. Dabei gibt es einen fließenden Übergang zwischen empirischer Forschung und Theorie, die als Interpretation oder Kritik konzipiert werden kann. [23]

Wir können also festhalten: In der amerikanischen Forschung hat die Krise der Repräsentation - anders als im deutschsprachigen Raum - die qualitative 
Forschung grundlegend verändert. Die damit verbundene Rezeption des Poststrukturalismus hat zu einer kritischen, ethisch engagierten und politisch orientierten Forschung geführt, die sich als ein neues Paradigma begreifen lässt. Weder Positivismus noch Postpositivismus leiten sie an. Es werden eigene Kriterien der Validität und der Legitimierung entwickelt, die sozialer Gerechtigkeit und einer radikalen progressiven Demokratie verpflichtet sind. Im folgenden möchte ich exemplarisch drei dieser Forschungsstrategien und -methoden näher diskutieren. [24]

\section{Strategien und Methoden einer kritischen qualitativen Forschung}

\subsection{Der interpretative Interaktionismus}

Norman DENZIN (1989, S.11) geht davon aus, dass es im sozialen Leben nur Interpretationen gibt, die zusammen mit Prozessen des Verstehens dessen wesentliche Merkmale sind. Er ist außerdem der Auffassung, dass jede interpretative Forschung mit der Biografie und dem Selbst des Forschers bzw. der Forscherin beginnt und endet (DENZIN 2009, S.108). Die eigenen Erfahrungen sollen in die Forschung einfließen und zu reichlich detaillierten und dichten Analysen führen. DENZIN (1989) forderte schon früh, die Sozialwissenschaften sollten sich problematischen und kritischen Lebenssituationen und -ereignissen zuwenden. Er beschäftigt sich vor allem mit den Erfahrungen und Erlebnissen, die den Sinn, den Menschen ihrem Leben verleihen, radikal verändern. Diese "Epiphanien", die unterschiedlich stark ausgeprägt sein können, nehmen unterschiedliche Gestalt an. Sie können sich plötzlich ereignen oder kumulativ vollziehen. Sie führen dazu, dass man gezwungen ist, sich selbst und den eigenen (Lebens-) Projekten neue Bedeutungen zu geben. Sie betreffen jeden Aspekt des Lebens und führen zu langfristigen und nicht umkehrbaren Veränderungen. [25]

Aufgabe der Forschenden ist es, diese Erfahrungen detailliert aufzuzeichnen, dicht zu beschreiben und sich den Geschichten zuzuwenden, die Menschen über den Verlust ihres Arbeitsplatzes, religiöse Konversionen, Scheidung, Vergewaltigung oder Gewalt in der Familie erzählen. SARTREs progressivregressiver Methode (1977) folgend, sollen Forschende das Subjekt historisch, zeitlich und sozial situieren. So soll untersucht werden, welche Bedingungen dazu geführt haben, dass das Subjekt diese Erfahrungen gemacht hat. Der Interpretationsprozess wendet sich anschließend den zukünftigen Folgen von Handlungen und Projekten zu. In der Untersuchung der interpretativen Materialien, die zu detailreichen kontextualisierten Beschreibungen verdichtet werden, sollen sowohl einzigartige Merkmale als auch Eigenschaften, die das Subjekt mit anderen teilt, zutage treten. Dabei ist das wesentliche Ziel des interpretativen Interaktionismus, darzulegen, wie sich historische und soziale Bedingungen im Leben miteinander interagierender Individuen auswirken. Diese selbst werden als universale Singularitäten begriffen, als Instanzen der universalen Themen, die die Postmoderne ausmachen, wie DENZIN (1989, S.139) feststellt. [26] 
Im Zentrum stehen die persönlichen Geschichten, die Menschen einander erzählen. Diese sollen dann kulturell und gesellschaftlich kontextualisiert werden, indem sie zu anderen geschriebenen Texten und Diskursen (z.B. Literatur, Film oder Fernsehen) in Beziehung gesetzt werden. Eine zentrale Frage ist, wie im Alltag interagierende Individuen ihr Leben mit Ideologien und Diskursen verknüpfen und in deren Licht ihre Erfahrungen interpretieren (vgl. DENZIN 1992, S.82). Hierbei ist ein wichtiger Interpretationsschritt, dass Forschende die in Texten und Diskursen gegebenen kulturellen Rahmen und Formen des Verstehens analysieren, die problematischen Lebenserfahrungen in einer Kultur Bedeutung geben. In der Postmoderne wird diesen vor allem durch ihre Darstellung in den Medien Bedeutung verliehen. Die Dekonstruktion kultureller Texte soll die wiederkehrenden Bilder und Vorstellungen eines Phänomens analysieren und dessen dominante Sinnrahmen bestimmen. In einem zweiten Schritt sollen subversive Lesarten der Texte bestimmt werden, die das Phänomen gegen den Strich lesen und neu interpretieren. Dieses Vorgehen soll zum einen helfen, Epiphanien besser zu verstehen, zum anderen sollen Forschende beim Schreiben ihrer Forschungstexte kulturelle Mythen, Ideologien oder Diskurse, die auch ihr Schreiben bestimmen, erkennen und im Gegenzug konkurrierende Modelle von "Wahrheit" und Interpretation aufzeigen. [27]

In der Postmoderne werden individuelle Probleme und kritische Lebensereignisse zu Waren, die auf dem Boulevard der Medien verkauft und zirkuliert werden. Sie werden zu öffentlichen Angelegenheiten, die betroffenen Personen zu Objekten. Dagegen möchte der interpretative Interaktionismus die Geschichten von Menschen, die von ihren existenziell problematischen, oft verborgenen Erlebnissen erzählen, in deren Sprache darstellen, sie emotional nachvollziehen und nach neuen Perspektiven für die Betroffenen suchen. [28]

DENZIN begreift seinen Ansatz als einen interpretativen Stil der Postmoderne, der Lesenden problematische Lebenserfahrungen in ihrer kulturellen und sozialen Kontextualisierung zugänglich machen möchte. Er zeigt, wie kulturelle Praktiken und Diskurse symbolische Expressionen und Geschichten prägen, aber auch, wie sich diese existenziellen Erfahrungen von den Erfahrungen anderer Betroffener unterscheiden. Er verwirklicht mit diesem Ansatz eine Forderung von Charles Wright MILLS, der in "The Sociological Imagination" (1959) dafür plädierte, dass die Soziologie untersuchen solle, wie private Probleme von Menschen mit öffentlichen Angelegenheiten und den öffentlichen Antworten auf diese Probleme zusammenhängen (vgl. DENZIN 2008a). MILLS war der Auffassung, dass eine soziologische Denkweise in der Postmoderne biografisch, interaktionistisch und historisch verankert sein solle. [29]

\subsection{Die Autoethnografie}

Die Autoethnografie ist eine Methode, die sich in der neueren amerikanischen Forschung großer Beliebtheit erfreut. Einen prominenten Vorläufer hat sie in den Arbeiten des Ethnologen und Schriftstellers Michel LEIRIS, insbesondere in seinem Werk "Phantom Afrika" (1980 [1934]), in dem er die ethnografische Methode auf seinen eigenen Alltag und seine Suche nach verborgenen Spuren 
des Heiligen anwendet. Eine Ethnografie der eigenen Erfahrung impliziert, dass der Forscher/die Forscherin sowohl Subjekt als auch Objekt ist. Hierbei können verschiedene methodologische Strategien zur Anwendung kommen: persönliche Erzählungen, Ich-Erzählungen, das Verfassen von Geschichten, Selbstbeobachtungen, kritische Autobiografie, biografische Methoden, evozierende Erzählungen oder die anthropologische Poetik. [30]

Ausgehend von der Krise der Repräsentation und der sie begleitenden Krise der Legitimation stellt sich auch in der Autoethnografie die Frage, wie in einer fragmentierten, flüchtigen und durch Sprache, Ideologien und Diskurse vermittelten Alltagswelt Geschichten erzählt werden und welchen Status sie haben können. Dabei wendet die Autoethnografie sich den persönlichen, konkreten und mundanen Details der Erfahrung zu, um Aufschluss über das Verhältnis von Selbst und Anderen, von Einzelnem und Gemeinschaft zu gewinnen (HOLMAN JONES 2005). Vor diesem Hintergrund definieren Carolyn ELLIS und Arthur BOCHNER (2003, S.214) Autoethnografien als Autobiografien: "(...) that self-consciously explore the interplay of the introspective, personally engaged self with cultural descriptions mediated through language, history, and ethnographic explanation" (siehe für einen Überblick ELLIS, ADAMS \& BOCHNER 2010). [31]

Gestützt auf den Poststrukturalismus wird in vielen Studien vor allem die Perspektivität, Partialität, Kontingenz und Begrenztheit biografischer Darstellungen hervorgehoben. Neue Schreibweisen und Aufführungen sind Antworten auf die Krise der Repräsentation. So können Prosastücke mit Musik, Tanz oder Theater verbunden werden, um die Leser/innen oder Zuschauer/innen anzusprechen. RONAI (1995) konfrontiert in ihrem Text "Multiple Reflections of Child Sex Abuse" Lesende mit unterschiedlichen, nebeneinandergestellten Reflexionen über den Missbrauch, den sie als Kind erleben musste. Sie benutzt eine geschichtete Darstellung, die mittels einer Vielfalt von Stimmen ihre traumatische Erfahrung hervorbringen und interpretieren lässt. Ihr Text macht deutlich, dass Autoethnografien Lesende berühren können und sollen sowie einen Raum für Reflexion, Diskussion und Dialog eröffnen, der zu einem Wandel von Einstellungen und Perspektiven führen kann. So sind Autoethnografien für subordinierte Personen und Gruppen eine gute Möglichkeit, sich zu artikulieren. Sie schließen hier eng an die Methode der testimonio an, eine Erzählung, die in der ersten Person über erlebte Gräueltaten wie Folter, über Gefängnisaufenthalte, aber auch über soziale Aufstände und den Kampf ums Überleben berichtet (vgl. BEVERLY 2005). [32]

Emotionale Erfahrungen spielen ebenfalls eine zentrale Rolle.

Autoethnografische Texte versuchen gerade, den körperlichen, sinnlichen und emotionalen Aspekt von Erlebnissen darzustellen. Sie zeigen, wie das Erzählen von Geschichten ein Selbst konstruiert. Dabei können Geschichten auch die Kontexte, in denen sie erzählt werden, verändern. Autoethnografien legen dar, wie Erzählungen uns helfen, unser Leben zu verstehen, zu interpretieren und zu verändern. Auf diese Weise werden die Notwendigkeit und die Macht von Erzählungen in unserem Leben hervorgehoben. Oft münden Autoethnografien in 
eine Performance, in eine Aufführung vor Publikum. Die intimen Geschichten, die situierte, prozesshafte, emotionale und intellektuelle Auseinandersetzungen mit dem Selbst darstellen, führen dann zu sehr persönlichen Begegnungen in einer weitgehend anonymen Öffentlichkeit (HOLMAN JONES 2005, S.773). Mit der Autoethnografie werden also demokratische Absichten verfolgt. Sie soll den Raum für Dialoge und Auseinandersetzungen erweitern mit dem Ziel, persönlichen und sozialen Wandel zu initiieren und zu unterstützen. [33]

\subsection{Performance ethnography}

Auch die performance ethnography ${ }^{1}$ ist eng mit einer demokratischen kulturellen Politik verknüpft (DENZIN 2008b; WINTER 2008). Sie lässt sich als eine Form ethnografischer Forschung begreifen, in deren Zentrum leiblich verankerte Interaktionen und deren Bedeutungen stehen. Der Ethnograf/die Ethnografin und bisweilen auch die von ihnen Untersuchten führen die Forschungsergebnisse vor Publikum auf. So kommt es zu einem gegenseitigen Austausch und Abgleichen von gelebten Erfahrungen, Emotionen, Perspektiven und Formen des Verstehens. Die Körper setzen Kultur in Bewegung, kommunizieren miteinander und führen zu einer intimen, manchmal leidenschaftlichen Konversation, die Machtstrukturen problematisiert und verändern möchte, um zur Selbstermächtigung beizutragen. Die Kultur und auch das Selbst werden als "Prozesse des Werdens" betrachtet, die in Aufführungen zum Ausdruck kommen. [34]

In seinem Essay "Rethinking Ethnography" (2006 [1991]) beschreibt Dwight CONQUERGOOD in einer analytisch subtilen Auseinandersetzung mit der ethnografischen Forschung die Merkmale und Bereiche einer kritischen performance ethnography sowie die performative Politik einer "verkörperten Untersuchung". Dabei plädiert er für eine radikale Neukonzeption des Untersuchungsprozesses, die er an folgenden Punkten festmacht. Im Anschluss an die postmoderne Ethnografie tritt er für eine "Rückkehr des Körpers" ein. Fast alle Ethnograf/innen würden in der Feldforschung erfahren, wie körperlich mühsam und anstrengend es sein könne, für eine bestimmte Zeit an einer Kultur teilzuhaben. Ein Verständnis einer fremden Kultur werde nicht nur kognitiv, sondern auch mit dem Leib erworben. So sei die Ethnografie "eine intensiv sinnliche Art der Erkenntnis" (CONQUERGOOD 2006 [1991], S.352), was in den publizierten ethnografischen Texten in der Regel nicht zum Ausdruck komme. Denn diese abstrahierten von den interpersonalen Kontingenzen des Feldes und den vielfältigen, oft beschwerlichen Erfahrungen. Stattdessen gehe es in den klassischen Texten um abstrakte Theorien, formale Analysen und Idealtypen. Der ethnografische Prozess umfasse aber unterschiedliche Praktiken, nicht nur das Beobachten, sondern auch das Sprechen, Hören und zusammen Handeln (S.353). Ethnograf/innen würden Wissen in den erfahrungsgesättigten und leiblich geprägten Interaktionen mit den Untersuchten erwerben, in denen die künstlichen Grenzen zwischen Beobachter/innen und Beobachteten verschwänden. Beide würden in ihrem Rollenspiel reziprok aufeinander reagieren und seien voneinander abhängig. Ethnograf/innen würden keinen unabhängigen

1 Zur Konzeption einer performativen Sozialwissenschaft vgl. FQS 9(2) (JONES et al. 2008). 
Beobachtungsplatz einnehmen, sondern sich einmischen, sie seien Teil der Forschung. Die Untersuchten, mit denen sie gemeinsam Zeit verbringen, seien ihre Zeitgenossen, auch wenn sie in ihren Berichten als "wild", "unterentwickelt", "subkulturell" oder "marginalisiert" beschrieben würden. CONQUERGOOD fordert eine neue Ethnografie, die ihre Schwerpunkte in den Praktiken des Sprechens, Zuhörens und temporären Zusammenlebens hat. So könne die textualistische Verengung der Ethnografie, ihr Textualismus, überwunden werden. [35]

CONQUERGOOD entfaltet auch ein postkoloniales Verständnis von Grenzen und Grenzziehungen, die sich als Differenzen in den postmodernen Subjekten selbst finden lassen, die jedoch nicht stabil, sondern durchlässig und überschreitbar seien (S.355ff.). Dies gelte auch für die Ethnografie selbst, die sich in Gebieten mit offenen Grenzen wiederfände und sich, wie ROSALDO (1989) zeigte, den borderlands, contact zones oder zones of difference zuwendet. Dies führe auch zu einer Neukonzeptualisierung von Identität und Kultur, deren Bedeutung nicht mehr ontologisch feststehe und stabil sei. Stattdessen seien sie kontingent, umkämpft, konstruiert und relational (CONQUERGOOD 2006 [1991], S.356). Es sind vor allem die postmodernen Erfahrungen von Reise, Migration, Flucht, Vertreibung und Exil, die Identität in etwas Provisorisches verwandeln, in eine immer wieder neue Aufführung mit fluidem, prozesshaftem Charakter. Gestrandet zwischen Welten schaffen entwurzelte und marginalisierte Menschen eine "erfinderische Poetik der Wirklichkeit" (CLIFFORD 1988, S.6). CONQUERGOOD (2006 [1991], S.357) bezieht sich auch auf "Kunst des Handelns" (1988) von Michel DE CERTEAU und dessen Analyse kreativen Vorgehens im Alltag (vgl. auch WINTER 2001b, S.197ff.) sowie auf seine eigene Feldarbeit mit Flüchtlingen und Migrant/innen im Gazastreifen, in Thailand und im Süden von Chicago. Auch hier macht er deutlich, dass Grenzen, Schwellen und Zwischenräume von Intensität geprägte Orte produktiver und kreativer kultureller Auseinandersetzung sein können. CONQUERGOOD (1998) bestimmt performance als eine Form der Überschreitung, die übernommene und sedimentierte Bedeutungen sowie normative Traditionen aufbricht, in Bewegung bringt und in politischen Auseinandersetzungen hegemoniale Strukturen infrage stellt. Performance wird für inn zu einem umfassenden und integrierenden Konzept, um kulturelle und soziale Prozesse verstehen und verändern zu können. [36]

Im Anschluss an CONQUERGOOD hat vor allem Norman DENZIN das Konzept einer performance ethnography in einen kritischen emanzipatorischen Diskurs überführt (vgl. WINTER 2001a, 2005). Er entwirft (vgl. DENZIN 2008b; WINTER \& NIEDERER 2008) eine interpretativ orientierte Ethnografie, die die Beobachter/innen als Interpret/innen versteht und sich Aufführungstexten zuwendet. So können im Feld geführte Interviews in zur Aufführung bestimmte Texte, in poetische Monologe oder in Stücke mit mehreren Personen transformiert werden. Sie zeigen, wie Menschen in sozialen Kontexten Geschichte schaffen und können die inspirierende Grundlage für die Transformation konkreter Situationen durch Akte der Neudefinition sein (vgl. DENZIN 2006, S.331). [37] 
In seiner Diskussion der grundlegenden Arbeiten von CONQUERGOOD weist DENZIN (1999) darauf hin, dass auch für den symbolischen Interaktionismus Kultur ein "Verb" sei und so als Prozess konzipiert werde. Darüber hinaus stünden Aufführungen und ihre Darstellungen im Zentrum gelebter Erfahrungen. Sie erwerben ihre Legitimität nicht durch das Zitieren wissenschaftlicher Texte, sondern dadurch, dass sie einen gemeinsamen Erfahrungsraum schaffen, in dem zwischen Aufführenden und Publikum Erlebnisse, Emotionen und Verständnisse geweckt, entfaltet und geteilt werden können. DENZIN (2003, S.9) bestimmt performance als einen "act of intervention, a method of resistance, a form of criticism, a way of revealing agency". Hierbei betont er, dass autoethnografische Zeugnisse eine wichtige Dimension der performance ethnography seien, weil sie soziale Missstände kritisieren, Kultur in Bewegung bringen und dem Publikum Erfahrung und Teilhabe ermöglichen. "As praxis, performance ethnography is a way of acting on the world in order to change it" (DENZIN 2006, S.331). [38]

Am Beispiel des Rassismus in den USA zeigt DENZIN (2008b) durch seine eigenen Forschungstexte und die Aufführungen, die er beschreibt, wie die Formen der Unterdrückung, aber vor allem der Widerstand gegen Rassismus und der Kampf für eine andere Welt Themen einer kritischen performativen Sozialwissenschaft sein können. Aufführungen können Empathie erzeugen, Verständnis bewirken und dazu beitragen, alternative soziale Wirklichkeiten zu kreieren. Das Ziel von Aufführungstexten ist es, einen kritischen und ethischen Diskurs zu initiieren und soziale Bindungen zu schaffen. Sie möchten nicht die Welt darstellen, wie sie "wirklich" ist, sondern intervenieren und ermächtigend wirken. Im Sinne von Richard RORTY (1989) geht es um den Wunsch nach Solidarität und deren gemeinsamer Realisierung. [39]

Es ist deutlich geworden, dass eine aufführungsorientierte Sozialwissenschaft durch eine kritische soziologische Imagination geprägt wird, die eine radikale Demokratisierung der Lebensverhältnisse anstrebt (vgl. DENZIN 2006, S.335). Während die postmoderne Sensibilität oft als zynisch, indifferent, pessimistisch, oberflächlich und instrumentell orientiert beschrieben wird, soll die Berücksichtigung der performativen Dimension zu Kritik, Widerstand, Hoffnung, Engagement, Solidarität und Freiheit führen. Sie reanimiert die kritische Theorie und zeigt, dass die scheinbar nicht überwindbare Eindimensionalität des Neoliberalismus eine hegemoniale Konstruktion ist. Dabei ist es Aufgabe einer kritisch orientierten Sozialwissenschaft, Erfahrung, Politik, Performativität und Ermächtigung miteinander zu verknüpfen. [40]

\section{Schluss}

Meine Ausführungen haben das Ziel gehabt zu zeigen, dass qualitative Forschung biografisch, kritisch und interventionistisch orientiert sein sollte. Wenn wir sie betreiben, befinden wir uns nicht in einem Machtvakuum. Auch hier gibt es hegemoniale Konstruktionen, die aber auch infrage gestellt werden können. Minoritäre Diskurse und Praktiken bringen neue Perspektiven hervor, die zu einer Neudefinition von Sinn und Relevanz qualitativer Forschung führen. Sie reagieren kreativ auf den problematischen Zustand einer "flüchtigen Moderne", auf die sich 
verschärfenden Formen sozialer und kultureller Ungleichheit im Neoliberalismus, auf die sich herausbildende Kontrollgesellschaft des Empire. Es scheint so, als müssten wir uns vom Mythos der wertfreien Wissenschaft nun endgültig verabschieden, so wie es Alvin GOULDNER (1962) schon vor langer Zeit gefordert hat, und als bliebe uns nichts anderes übrig als zu erkennen, dass auch Wissenschaft eine politische Praxis ist und dass wir uns, wie Howard BECKER schon 1967 festgestellt hat, für eine Seite entscheiden müssen. [41]

\section{Literatur}

Baudrillard, Jean (1982). Der symbolische Tausch und der Tod. München: Matthes \& Seitz. Baudrillard, Jean (1983). Simulations. New York: Semiotext.

Bauman, Zygmunt (2005). Afterthougt: On writing: On writing sociology. In Norman K. Denzin \& Yvonna S. Lincoln (Hrsg.), The Sage handbook of qualitative research (3. Aufl., S.1089-1098). London: Sage.

Beck, Ulrich (2007). Weltrisikogesellschaft. Frankfurt/M.: Suhrkamp.

Becker, Howard S. (1967). Whose side are we on? Social Problems, 14, 239-247.

Beverley, John (2005). Testimonio, subalternity, and narrative authority. In Norman K. Denzin \& Yvonna S. Lincoln (Hrsg.), The Sage handbook of qualitative research (3. Aufl., S.547-558). London: Sage.

Bourdieu, Pierre et al. (1997). Das Elend der Welt. Konstanz: UVK.

Bruner, Edward M. (1986). Experience and its expressions. In Victor M. Turner \& Edward M. Bruner (Hrsg.), The anthropology of experience (S.3-30). Urbana: University of Illinois Press.

Castoriadis, Cornelius (1984). Gesellschaft als imaginäre Institution. Entwurf einer politischen Philosophie. Frankfurt/M.: Suhrkamp.

Clifford, James (1988). The predicament of culture. Cambridge, MA: Harvard University Press.

Conquergood, Dwight (1998). Beyond the text. Towards a performative cultural politics. In Sam J. Dailey (Hrsg.), The future of performance studies: Visions and revisions (S.25-36). Washington: National Communication Association.

Conquergood, Dwight (2006/1991). Rethinking ethnography: Towards a critical cultural politics. In D. Soyini Madison \& Judith Hamera (Hrsg.), The Sage handbook of performance studies (S.351-366). London: Sage.

De Certeau, Michel (1988). Kunst des Handelns. Berlin: Merve.

Denzin, Norman K. (1989). Interpretive interactionism. London: Sage.

Denzin, Norman K. (1991). Images of postmodern society. Social theory and contemporary cinema. London: Sage.

Denzin, Norman K. (1992). Symbolic interactionism and cultural studies. The politics of interpretation. Oxford, CA: Blackwell.

Denzin, Norman K. (1997). Interpretive ethnography. Ethnographic practices for the 21st century. London : Sage.

Denzin, Norman K. (1999). Ein Schritt voran mit den Cultural Studies. In Karl H. Hörning \& Rainer Winter (Hrsg.), Widerspenstige Kulturen. Cultural Studies als Herausforderung (S.116-145). Frankfurt/M.: Suhrkamp.

Denzin, Norman K. (2003). Performance ethnography. Critical pedagogy and the politics of culture. London: Sage.

Denzin, Norman K. (2006). The politics and ethics of performance ethnography. Toward a pedagogy of hope. In D. Soyini Madison \& Judith Hamera (Hrsg.), The Sage handbook of performance studies (S.325-337). London: Sage.

Denzin, Norman K. (2008a). Programmatische Ansprache des Vorsitzenden: Ein neuer Blick auf C. Wright Mills' The Sociological Imagination. In Rainer Winter \& Elisabeth Niederer (Hrsg.), 
Ethnographie, Kino und Interpretation - die performative Wende der Sozialwissenschaften. Der Norman K. Denzin Reader (S.11-48). Bielefeld: transcript.

Denzin, Norman K. (2008b). Ein Plädoyer für die performative Dimension. In Rainer Winter \& Elisabeth Niederer (Hrsg.), Ethnographie, Kino und Interpretation - die performative Wende der Sozialwissenschaften. Der Norman K. Denzin Reader (S.169-202). Bielefeld: transcript.

Denzin, Norman K. (2009). Qualitative inquiry under fire. Toward a new paradigm dialogue. Walnut Creek, CA: Left Coast Press.

Denzin, Norman K. \& Lincoln, Yvonna S. (2005). Introduction: The discipline and practice of qualitative research. In Norman K. Denzin \& Yvonna S. Lincoln (Hrsg.), The Sage handbook of qualitative research (3. Aufl., S.1-32). London: Sage.

Derrida, Jacques (1988). Randgänge der Philosophie. Wien: Passagen Verlag.

Devereux, George (1973). Angst und Methode in den Verhaltenswissenschaften. München: Hanser.

Ellis, Carolyn \& Bochner, Arthur P. (2003). Autoethnography, personal narrative, reflexivity. In Norman K. Denzin \& Yvonna S. Lincoln (Hrsg.), Collecting and interpreting qualitative materials (S.199-258). London: Sage.

Ellis, Carolyn; Tony E. Adams \& Bochner, Arthur P. (2010). Autoethnography. Forum Qualitative Sozialforschung / Forum: Qualitative Social Research, 12(1), Art. 10, http://nbnresolving.de/urn:nbn:de:0114-fqs1101108.

Fiske, John (1999). Wie ein Publikum entsteht. Kulturelle Praxis und Cultural Studies. In Karl H. Hörning \& Rainer Winter (Hrsg.), Widerspenstige Kulturen. Cultural Studies als Herausforderung (S.238-263). Frankfurt/M.: Suhrkamp.

Gouldner, Alvin (1962). Anti-Minotaur. The myth of a value-free sociology. Social Problems, 9, 199213.

Haraway, Donna (1997).Modest_witnesses@second_millenium.FemaleMan_Meets-OncoMouse. feminism and technoscience. London: Routledge.

Haraway, Donna (2004/1991). Situated knowledges: The science question in feminism and the privilege of partial perspective. In Sandra Harding (Hrsg.), The feminist standpoint theory reader. Intellectual and political controversies (S.81-102). London: Routledge.

Harding, Sandra (2004/1988). Rethinking standpoint epistemology: What is "strong objectivity". In Sandra Harding (Hrsg.), The feminist standpoint theory reader. Intellectual and political controversies (S.127-140). London: Routledge.

Holman Jones, Stacy (2005). Autoethnography: Making the personal political. In Norman K. Denzin \& Yvonna S. Lincoln (Hrsg.), The Sage handbook of qualitative research (3. Aufl., S.763-792). London: Sage.

Jones, Kip; Gergen, Mary; Yallop, J. Guiney; Lopez de Vallejo, Irene; Roberts, Brian \& Wright, Peter (Hrsg.) (2008). Performative Sozialwissenschaft. Forum Qualitative Sozialforschung / Forum: Qualitative Social Research, 9(2), http://www.qualitative-research.net/index.php/fqs/issue/view/10.

Kellner, Douglas (1995). Media culture. London: Routledge.

Lather, Patti (2001 [1993]). Fertile obsesssion: Validity after poststructuralism. In Norman K. Denzin \& Yvonna S. Lincoln (Hrsg.), The American tradition in qualitative research (Bd. 4; S.29-52). London: Sage.

Lather, Patti (2003/1986). Issues of validity in openly ideological research. Between a rock and a soft place. In Yvonna S. Lincoln \& Norman K. Denzin (Hrsg), Turning points in qualitative research. Tying knots in a handkerchief (S.185-215). Walnut Creek, CA: Altamira Press.

Leiris, Michel (1980/1934). Phantom Afrika. Teil 1. Frankfurt/M.: Syndikat.

McLaren, Peter (2001). Che Guevara, Paolo Freire and the pedagogy of revolution. Boulder, CO: Rowman \& Littlefield.

Mills, Charles Wright (1959). The sociological imagination. New York: Oxford University Press.

Niederer, Elisabeth (2009). Die Kultur der Armut und ihre sozialen Kontexte. Eine ethnographische Studie zu Armutslagen in Kärnten. Dissertation, Fakultät Kulturwissenschaften der Alpen-Adria Universität Klagenfurt.

Richardson, Laurel (2003). Writing: A method of inquiry. In Norman K. Denzin \& Yvonna S. Lincoln (Hrsg.), Collecting and interpreting qualitative materials (S.499-541). London: Sage. 
Ronai, Carol R. (1995). Multiple reflections of childhood sex abuse. An argument for a layered account. Journal of Contemporary Ethnography, 23(4), 395-426.

Ronai, Carol R. (1998). Sketching with Derrida: An ethnography of a researcher/erotic dancer. Qualitative Inquiry, 4(3), 405-420.

Ronai, Carol R. (1999). The next night sous rature: Wrestling with Derrida's mimesis. Qualitative Inquiry, 5(1), 114-129.

Rorty, Richard (1989). Kontingenz, Ironie und Solidarität. Frankfurt/M.: Suhrkamp.

Rosaldo, Renato (1989). Culture and truth: The remaking of social analysis. Boston: Beacon.

Sartre, Jean-Paul (1977). Der Idiot der Familie, Bd. 1. Reinbek: Rowohlt.

Smith, Dorothy (2004 [1974]). Women's perspective as a radical critique of sociology. In Sandra Harding (Hrsg.), The feminist standpoint theory reader. Intellectual and political controversies (S.21-34). London: Routledge.

Trinh, T. Minh-ha (1989). Woman, native, other: Writing, postcoloniality and feminism. Bloomington: Indiana University Press.

Winter, Rainer (2001a). Ethnographie, Interpretation und Kritik. Aspekte der Methodologie der Cultural Studies. In Udo Göttlich, Lothar Mikos \& Rainer Winter (Hrsg.), Die Werkzeugkiste der Cultural Studies. Perspektiven, Anschlüsse und Interventionen (S.43-62). Bielefeld: transcript.

Winter, Rainer (2001b). Die Kunst des Eigensinns. Cultural Studies als Kritik der Macht. Weilerswist: Velbrück Wissenschaft.

Winter, Rainer (2005). Interpretative Ethnographie. In Lothar Mikos \& Claudia Wegener (Hrsg.), Qualitative Medienforschung. Ein Handbuch (S.553-561). Konstanz: UVK.

Winter, Rainer (2006). Kultur, Reflexivität und das Projekt einer kritischen Pädagogik. In Paul Mecheril \& Monika Witsch (Hrsg.), Cultural Studies und Pädagogik. Kritische Artikulationen (S.21-50). Bielefeld: transcript.

Winter, Rainer (2008). Die Politik der Aufführung. Interpretative Ethnographie und kritische Pädagogik im 21. Jahrhundert. In Johannes Fromme \& Werner Sesink (Hrsg.), Pädagogische Medientheorie (S.115-128). Wiesbaden: VS Verlag.

Winter, Rainer (2010). Widerstand im Netz. Zur Herausbildung einer transnationalen Öffentlichkeit durch netzbasierte Kommunikation. Bielefeld: transcript.

Winter, Rainer \& Niederer, Elisabeth Niederer (Hrsg.) (2008). Ethnographie, Kino und Interpretation - die performative Wende der Sozialwissenschaften. Der Norman K. Denzin Reader. Bielefeld: transcript.

Zima, Peter V. (2007). Kritische Theorie als Dialogische Theorie. In Rainer Winter \& Peter V. Zima (Hrsg.), Kritische Theorie heute (S.97-114). Bielefeld: transcript.

\section{Zum Autor}

Rainer WINTER ist Soziologe und Psychologe. Seit 2001 ist er Professor für Kultur- und Medientheorie an der Alpen-Adria-Universität in Klagenfurt am Wörthersee.
Kontakt:

Univ.-Prof. Dr. Rainer Winter

Alpen-Adria-Universität Klagenfurt Institut für Medien- und Kommunikationswissenschaft Universitätsstraße 65-67 9020 Klagenfurt, Österreich

Tel.: 004346327001827

Fax.: 004346327001899

E-Mail: Rainer.Winter@uni-klu.ac.at URL: http://rainer-winter.net/

\section{Zitation}

Winter, Rainer (2010). Ein Plädoyer für kritische Perspektiven in der qualitativen Forschung [41 Absätze]. Forum Qualitative Sozialforschung / Forum: Qualitative Social Research, 12(1), Art. 7, http://nbn-resolving.de/urn:nbn:de:0114-fqs110171. 